\title{
A novel porcine model of early left ventricular dysfunction for translational research
}

This article was published in the following Dove Press journal:

Research Reports in Clinical Cardiology

24 January 2013

Number of times this article has been viewed

\section{Nadim Malik \\ Kelly A Farrell \\ Sarah B Withers \\ Elizabeth J Wright \\ Cathy M Holt}

Institute for Cardiovascular Science, University of Manchester, Manchester, United Kingdom
Correspondence: Cathy M Holt Institute for Cardiovascular Science, University of Manchester, 3.3 Ib Core Technology Facility, 46 Grafton St, Manchester, MI 3 9NT, United Kingdom Tel +44 I6। 275 567|

Fax +44 |6| 275 | I83

Email cathy.holt@manchester.ac.uk
Background: The early stages of left ventricular (LV) dysfunction account for a much larger proportion of the population with heart disease than that with clinical heart failure. However, LV dysfunction is more difficult to diagnose than established heart failure, and because of this it is not usually treated. Research on LV dysfunction is commonly conducted in small animal models in which the cardiac pathophysiology is dissimilar to that in humans, thereby restricting translation. This study aimed to use a novel pig model of mild to moderate early ischemic LV dysfunction to assess the effects of such dysfunction in the myocardium.

Methods: Multiple areas of controlled microinfarcts were created via microembolization using embolization beads, with invasive hemodynamic and transthoracic echocardiographic assessment of LV function. Four weeks after intervention, the hearts were explanted for determination of the infarcted surface area and analysis of calcium regulatory proteins.

Results: In vivo hemodynamic measurements confirmed a $>25 \%$ decrease in $\mathrm{LV} \mathrm{dP} / \mathrm{dt}$ (maximum and minimum) with creation of microinfarcts compared with baseline, whilst echocardiography showed mild to moderate LV dysfunction. Perioperative mortality was $10 \%-15 \%$. In surviving pigs, morphometry at 4 weeks confirmed that up to $20 \%$ of the total LV surface area contained microinfarcts. Western blot analysis showed alterations in levels of the calcium regulatory proteins, sarcoplasmic reticulum $\mathrm{Ca}^{2+}$ ATPase and sodium-calcium exchange, in infarcted areas, compared with normal LV tissue from the same animals.

Conclusion: These results demonstrate the usefulness of this model for investigation of the precise molecular and cellular changes associated with early mild to moderate LV dysfunction from ischemic injury, and its potential use for modulating these changes with the aim of achieving functional reversibility or regeneration of the myocardium.

Keywords: left ventricular dysfunction, heart failure, porcine model

\section{Introduction}

Over the last decade, use of disease-modifying therapies (angiotensin-converting enzyme inhibitors, angiotensin II receptor antagonists, beta-blockers, and aldosterone antagonists) has led to some improvements in the morbidity and mortality associated with heart failure. However, most of these therapies aim to modulate the neurohumoral pathway. Research into the molecular mechanisms involved, using mainly small animal models, has identified the importance of calcium dysregulation in the pathogenesis and progression of heart failure. ${ }^{1-3}$ However, translational clinical application is often limited, mostly because of the dissimilarities in the pathophysiology between these models and humans. Some research is now being performed in large animal models, where the pathophysiology is more similar to that of humans. However, most current submit your manuscript $\mid$ www.dovepress.com

Dovepress

http://dx.doi.org/10.2147/RRCC.\$38656
Research Reports in Clinical Cardiology 2013:4 I-7

(C) 2013 Malik et al, publisher and licensee Dove Medical Press Ltd. This is an Open Access article which permits unrestricted noncommercial use, provided the original work is properly cited. 
large animal models of heart failure use either pacing or surgical ligation of an artery to inflict clinically advanced heart failure. For ischemic left ventricular (LV) dysfunction, three different modalities may be employed, ie, induction of a single large myocardial infarction (MI) due to arterial occlusion, eg, the circumflex artery occlusion model, ${ }^{4,5}$ chronic coronary artery stenosis with surgical placement of aneroid bands allowing reversible hibernation/stunning-related LV dysfunction, and LV dysfunction due to widespread microemboli/ microinfarcts. ${ }^{6}$ Other models of LV dysfunction have been described, such as diastolic dysfunction secondary to renal hypertension. ${ }^{7}$ Therefore, current models usually have clinically advanced LV dysfunction with debatable etiological significance, ie, representing only a fraction of the clinically relevant pathology, such as single artery occlusion or ligation, rapid ventricular pacing, and injection of cardiotoxic agents. To date, only the sheep model features widespread ischemic injury and loss of myocardium, which is one of the most common forms of clinical heart failure, to induce such LV impairment. ${ }^{8,9}$ However, this too results in establishment of clinically advanced heart failure. Moreover, the pathophysiology of the sheep model is yet to be characterized fully.

We aimed to establish a reliable, reproducible, and temporally stable pig model of mild to moderate early ischemic LV dysfunction by inflicting multiterritory microinfarcts using the technique of microembolism. Use of the pig model for cardiovascular research is already well characterized. ${ }^{10-14}$ This model has recently been used for translational research by offering insights into the pathophysiology of early dysfunction and analysis of developing therapies aimed at preventing progression to established advanced heart failure whilst also allowing for translational "bench-to-bedside" research in early heart failure. ${ }^{15}$

\section{Materials and methods}

All animal experiments outlined in this work were performed according to current UK Home Office regulations and with the approval of the relevant University of Manchester local ethics committee. All the work was overseen by a trained veterinary surgeon as per the institution's regulations. Premedication included aspirin $75 \mathrm{mg}$ /day for 48 hours, and this was continued for the duration of the study.

\section{Anesthesia and perioperative monitoring}

The pigs were not fed for 24 hours prior to the procedure. Anesthesia was induced by inhalation (halothane 3\%-4\%) only. Each animal was placed in the appropriate position, intubated, anesthetized with a mixture of $1.5 \%$ halothane
(Trofiels, Zug, Switzerland) and 98.5\% oxygen (CFPO, Paris, France), and ventilated for the duration of the procedure. End-tidal $\mathrm{CO}_{2}$ levels were measured continuously and maintained between $26 \mathrm{mmHg}$ and $36 \mathrm{mmHg}$ with a monitor (N1000, Nellcor, Pleasanton, California). Peripheral arterial oxygen saturation, maintained at a level higher than $95 \%$, was monitored with an appropriate ear probe. An electrocardiogram was used to monitor each animal continuously during the procedure, and transthoracic echocardiography was performed before and after each embolization procedure.

\section{Preparation of beads/microspheres for embolization}

Commercially available embolization beads/microspheres (200-500 $\mu \mathrm{m}$ diameter) were supplied sterile in syringes. Beads were prepared as per the manufacturer's protocol (Biocompatibles Ltd, Farnham, UK) and $0.25 \mathrm{~mL}$ of the solution was initially diluted with heparinized Ringer's lactate solution to a total volume of $1 \mathrm{~mL}$, which was then agitated for a few minutes. Thereafter, $0.1 \mathrm{~mL}$ of this solution was drawn into a $1 \mathrm{~mL}$ syringe and diluted to a $1 \mathrm{~mL}$ volume with Ringer's lactate solution immediately prior to intracoronary infusion. The volumes of the 200-500 $\mu \mathrm{m}$ diameter beads used for initial experimentation to determine a sublethal outcome were $1000 \mu \mathrm{L}, 500 \mu \mathrm{L}, 300 \mu \mathrm{L}, 200 \mu \mathrm{L}$, and $100 \mu \mathrm{L}$ doses of microspheres.

\section{Creation of MI to establish LV dysfunction}

Twenty-four white Yorkshire pigs (14-18 kg) underwent embolization procedures to determine a suitable dose for achieving MI. A right carotid artery puncture was performed and a 6-8 French vascular sheath was put in place. Embolization experiments were carried out on the right coronary $(n=3)$, left anterior descending $(n=3)$, diagonal $(n=3)$, circumflex $(n=3)$, and obtuse marginal $(n=3)$ arteries. Following embolization with an optimal sublethal dose $(200 \mu \mathrm{L})$, the end point of such initial experimentation was defined as survival with recovery to 4 weeks. Thereafter, selective catheterization of the left anterior descending artery was performed using a guide catheter, and a coronary angiogram was obtained prior to placement of an angioplasty wire and catheter $(n=6)$. Selective embolization of the beads/microspheres was then performed in order to create microinfarctions, as guided by the electrocardiogram, transthoracic echocardiogram, and invasive hemodynamic measurements. Finally, an angiogram was repeated before the end of experimentation to confirm that the major epicardial arteries were unobstructed, and an appropriate dose of 
analgesia was administered (2.5 $\mathrm{mg}$ buprenorphine). At the end of the experiment, all equipment was removed, hemostasis was secured, and the animals were allowed to recover fully for the planned duration of the experiment.

\section{Echocardiographic assessment of $L V$ function}

A transthoracic echocardiograph machine (Accuson, Siemens, Johannesburg, South Africa) and probe were used to quantify the degree of acute mild LV dysfunction (at the time of embolism/infarction), compared with baseline (normal) function. General Electric Echopack software for Windows 2008 (General Electric, Louisville, KT) was used to analyze the LV areas in apical long axis views to calculate LV ejection fraction (EF) and therefore LV function. Echocardiographically moderate LV dysfunction was defined as an EF (LVDA-LVSA/LVDA $\times 100)$ of $30 \%-40 \%$, with an EF of $40 \%-50 \%$ defined as mild LV dysfunction (LVDA, left ventricular diastolic area; LVSA, left ventricular systolic area). LV function was also reassessed at 4 weeks.

\section{Animal welfare}

The overall health and well-being of each animal was monitored daily for clinical signs of heart failure until the end of the study. Prior to euthanasia, transthoracic echocardiography was repeated on each animal. The animals were sacrificed at 4 weeks post procedure by lethal injection with intravenous pentobarbital (18\% solution). After excision, the whole heart was placed in a kidney dish containing sterile normal saline for immediate assessment.

\section{Pathological and histological evaluations Gross pathology}

The presence of epicardial area(s) of infarction was identified in each explanted heart. The gross pathology of the heart was photographed, and semiquantitative morphometry was subsequently performed. For comparative analysis, the total infarction area was represented as a percentage of the total area of the LV free wall which was visible. Thereafter, the heart was dissected and the three areas of interest in the LV free wall were identified as the LV apex, mid LV, and base of the LV. For each area, the presence and absence of MI was identified and 5-10 mm blocks of full thickness tissue samples were dissected. LV myocardial tissue samples were then processed for histology or snapfrozen for protein analysis. For each of these samples, a description was provided, including full thickness MI, partial thickness MI, or normal myocardium.

\section{Preparation of histological sections} for hematoxylin and eosin staining

The specimens were fixed in 10\% neutral buffered formalin for 24 hours, dehydrated in a series of alcohols, set in xylene, embedded in paraffin, and 3-4 $\mu \mathrm{m}$ sections were cut for microscopic analysis. Each section was stained with hematoxylin and eosin and examined under a light microscope (Leica DM5000).

\section{Assessment of calcium regulatory proteins}

Tissue was obtained from at least three areas within the LV myocardial free wall, ie, areas with infarction/microinfarcts, areas surrounding the infarction/microinfarcts (border zone), and distant areas. Samples were immediately snapfrozen. Western blot analysis was performed to investigate differences in expression levels for various calcium regulatory proteins, ie, sarcoplasmic reticulum calcium ATPase (MA3-919, Affinity Bioreagents, Golden, CO), sodium-calcium exchanger (11-13, Swant, Marly, Switzerland), phospholamban (05-205, Millipore, Billerica, MA), calcineurin (556350, BD Pharmingen, San Diego, CA), and calsequestrin (06-382, Millipore). A goat anti-mouse horseradish peroxidase secondary antibody (P0447, Dako, Fort Collins, CO) was used to visualize bands. Bands were quantified by densitometry using Quantity One software (Bio-Rad Laboratories, Hemel Hempstead, UK) and normalized to GAPDH (8-GAPDH-h, Advanced ImmunoChemical, Long Beach, CA).

\section{Statistical analysis}

The results are expressed as the mean \pm standard error of the mean. The Student's $t$-test or one-way analysis of variance with the Bonferroni post hoc test for multiple comparisons was performed. Statistical significance is denoted as follows: $* P<0.05, * * P<0.01, * * * P<0.001$.

\section{Results \\ Induction of sublethal MI}

Initial experiments ( $\mathrm{n}=24$ pigs) were aimed at identifying the sublethal dose of microspheres that could be used in the porcine coronary circulation. The $300-1000 \mu \mathrm{L}$ doses resulted in more than $80 \%$ mortality within 30 minutes of infarction. Only use of the $200 \mu \mathrm{L}$ and $100 \mu \mathrm{L}$ doses of microspheres was not associated with a lethal outcome ( $<15 \%$ mortality), and use of the $100 \mu \mathrm{L}$ dose of microspheres was associated with little clinical evidence of MI ( $0 \%$ mortality). We confirmed that a $200 \mu \mathrm{L}$ dose of microspheres was sufficient to induce sublethal infarcts 
without occluding the major epicardial coronary arteries. In addition, such experimentation also identified the diagonal and obtuse marginal branches of the left coronary system as suitable for induction of sublethal $\mathrm{MI} / \mathrm{microinfarcts}$ in the LV muscle, whilst the injections in the branches of the right coronary artery did not show any $\mathrm{MI} / \mathrm{microinfarcts}$ in the LV muscle.

In spite of the above initial experimentation, which clarified the dose of microspheres required and the relevant coronary artery branches to achieve reproducible but sublethal $\mathrm{MI} /$ microinfarcts, the mortality rate related to actual MI remained at $10 \%-15 \%$ in all the subsequent experimentation.

\section{Mild to moderate ischemic LV dysfunction}

Thirty minutes after induction of MI, transthoracic echocardiographic assessment confirmed a decrease in estimated LVEF $(P<0.001$ compared with pre-MI LVEF, $\mathrm{n}=6$ ). LV function was confirmed to be still in the range of mild to moderate impairment compared with baseline. Assessment of EF repeated at 4 weeks after induction of MI showed results similar to those obtained at 30 minutes post-infarction (Figure 1). The percentage difference in EF post-MI compared with baseline EF was in the range of $17 \%-18 \%$.

\section{In vivo hemodynamic assessment of $\mathrm{dP} / \mathrm{dT}$ after induction of $\mathrm{MI}$}

Hemodynamic measurements 30 minutes after onset of MI confirmed a more than $25 \%$ decrease in $\mathrm{LV} \mathrm{dP} / \mathrm{dT}$ (maximum and minimum) compared with baseline (Figure 2).

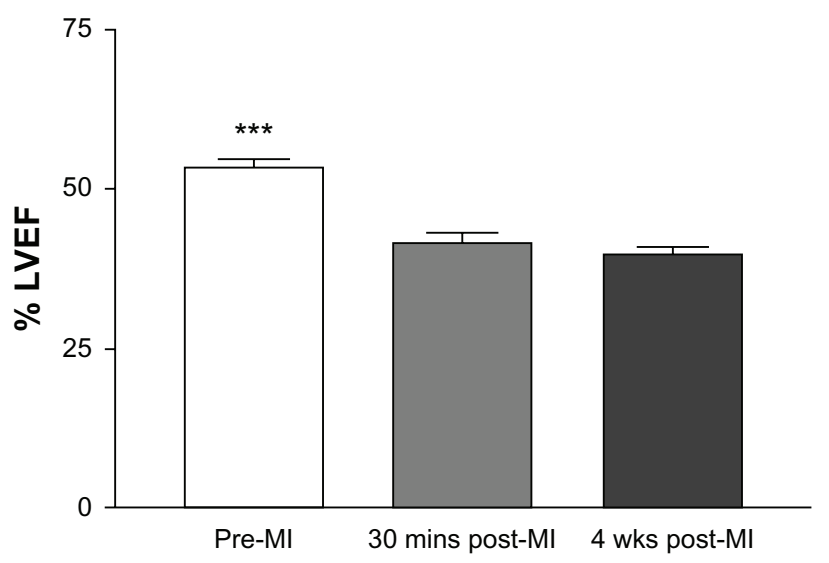

Figure I Transthoracic echocardiographic assessment of LVEF, showing mild to moderate reduction in ejection fraction 30 minutes after $\mathrm{MI}$ injury, compared with baseline ejection fraction (pre-MI injury), and ejection fraction at four weeks post-MI injury. $* * *(P<0.00 I)$.

Abbreviations: LVEF, left ventricular ejection fraction; MI, myocardial infarction.

\section{Histomorphometric assessment of MI}

Gross examination confirmed the presence of MI/microinfarcts (Figure 3A and B). Mean total infarct area, expressed as a percentage of total LV surface area, was calculated as $19.4 \% \pm 6.5 \%(n=6)$. Histological assessment by hematoxylin and eosin staining showed areas of microinfarcts adjacent to areas of normal myocardium (Figure 3C).

\section{Assessment of calcium regulatory proteins}

Histological analysis of sections stained with hematoxylin and eosin revealed an absence of tissue reaction to the beads. Western blot analysis of calcium regulatory proteins showed a decrease in expression of sarcoplasmic reticulum $\mathrm{Ca}^{2+}$ ATPase, calsequestrin, and sodium-calcium exchanger ( $80 \mathrm{kDa}$ isoform) in full thickness infarct tissue from the LV when compared with normal LV. There was no change in expression of phospholamban and calcineurin in the infarcted areas compared with normal myocardium obtained from the base of the heart (Figure 4).

\section{Discussion}

We have successfully refined the reliability, reproducibility, and temporal stability of a pig model of mild to moderate early ischemic LV dysfunction by inflicting infarcts/microinfarcts using the technique of multiterritory microembolism. The results reported here indicate changes in calcium regulatory proteins relating to early $\mathrm{LV}$ dysfunction, with characterization of these changes in the areas of MI. Overall, these results demonstrate the usefulness of this model for investigation of precise molecular and cellular changes associated with early mild to moderate LV dysfunction from ischemic injury, and its potential use for modulating these changes with the aim of achieving functional reversibility in the myocardium.

\section{Use of current model of HF}

Having successfully established a reliable and reproducible pig model of early mild to moderate LV dysfunction using a microinfarction technique, this model will be useful for investigating molecular, cellular, and functional changes in early reversible LV dysfunction. It is predicted that the currently described model will offer insights into novel calcium-related and other cellular mechanisms involved in early LV dysfunction and what precisely within the myocardium may determine functional/structural recovery. The model will allow more clinically relevant pathophysiological translational research helping to answer basic but important questions such as, do specific therapies in current use help 


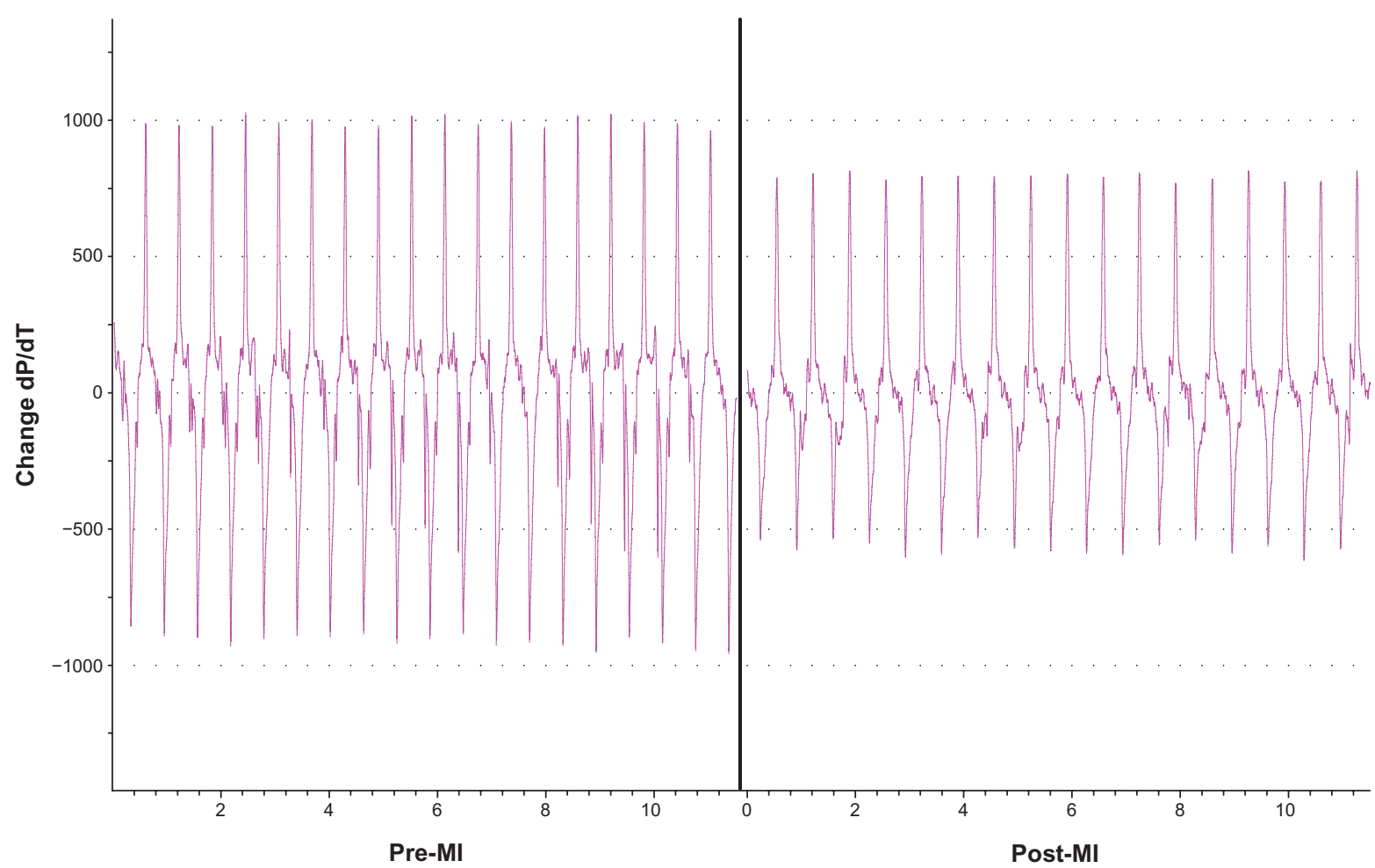

Figure 2 Invasive hemodynamic changes in $\mathrm{dP} / \mathrm{dT}$, pre-MI injury (tracing on the left) and 30 minutes post-Ml injury (tracing on the right), confirming a $25 \%$ reduction in left ventricular $\mathrm{dP} / \mathrm{dT}$, in keeping with mild to moderate LV dysfunction.

Abbreviation: Ml, myocardial infarction.

to achieve permanent or temporary reversibility in dysfunction (ie, are there implications of stopping these drugs after a short period?), and are angiotensin-converting enzyme inhibitors and beta-blockers required together or does each alone confer sufficient reversibility of dysfunction? Other potentially longer-term applications of this model would
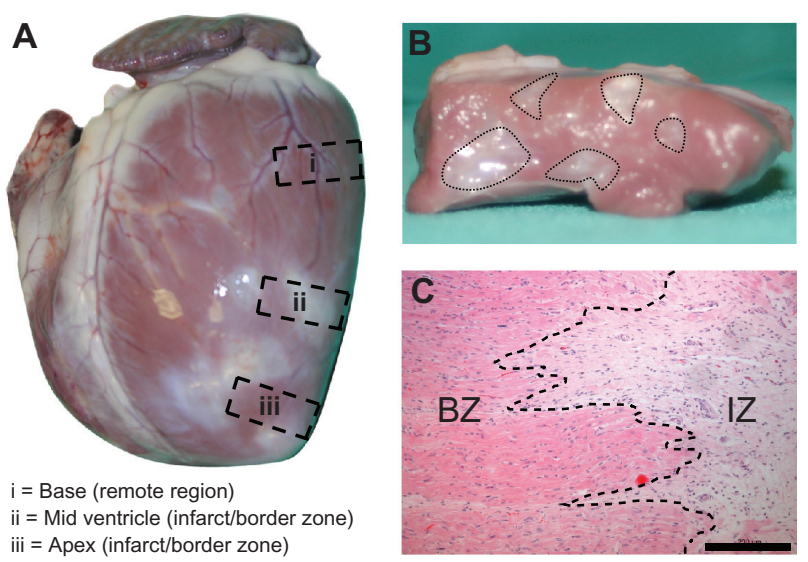

Figure 3 (A) Photographic representation of tissue samples obtained from porcine hearts, with areas marked as myocardial infarction zone in the apex (iii), border zone (ii), and distant zone in the base (i). (B) Cross-section of porcine ventricular wall 4 weeks post-infarct. Microinfarcts are visible in several areas of the left ventricular free wall. (C) Hematoxylin and eosin staining shows the clear distinction between infarct (IZ) and border (BZ) tissue. include genetic manipulations of the calcium dysregulation processes and/or use of appropriate stem cell therapies for reversing LV dysfunction. Indeed, recent work in our laboratory has used the model described in this study to investigate the effects of encapsulated genetically modified mesenchymal stromal cells on myocardial recovery following MI. ${ }^{15}$

Likewise, unlike the model of large MI due to occlusion of a large epicardial artery, the potential advantage of using the microinfarction technique with multiple but small MIs would be to assess the hypothesis of any potential paracrine benefits derived from corrective therapies aimed at regenerating or repairing the myocardium via such strategies. This might be particularly relevant for gene therapies and/or stem cell therapies delivered at the site of MI.

The current model is applicable only to ischemic heart failure and not to other forms of heart failure. It also has the limitation that in this model the only diseased artery with associated myocardial injury/damage is the index artery territory and the other two are normal, unlike clinical cases where multiterritory ischemia/infarction leading to LV dysfunction involving two or three main arteries is more common. 
A

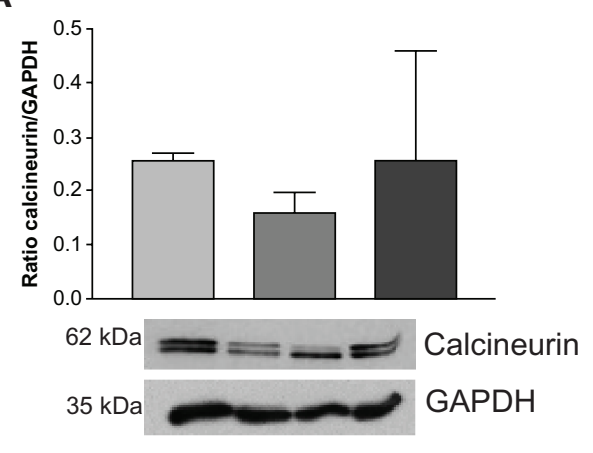

$\mathrm{N}$ PT FT C

B

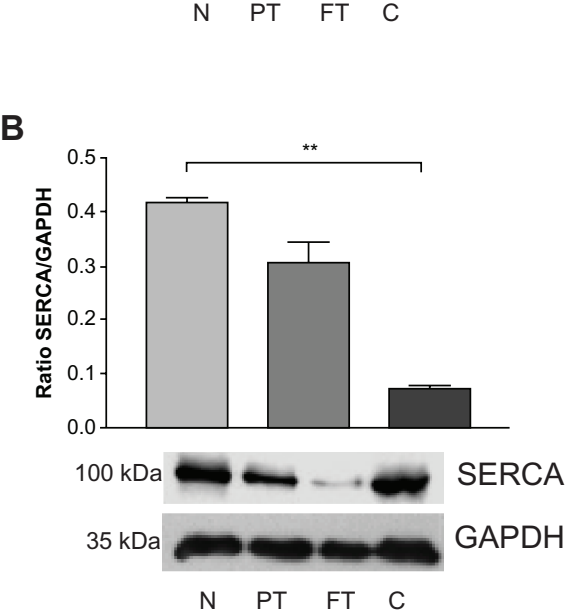

D

C

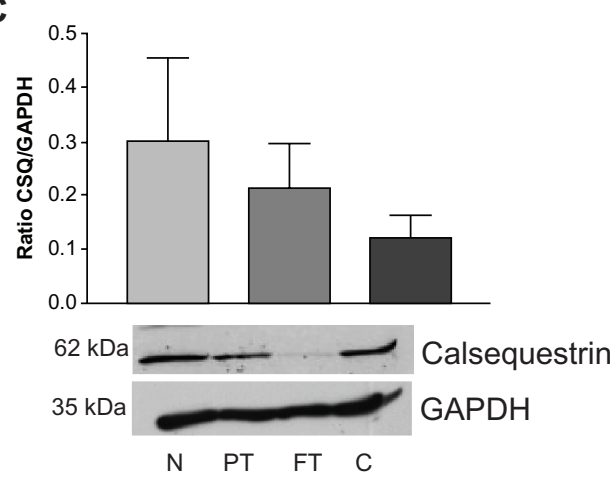

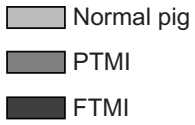
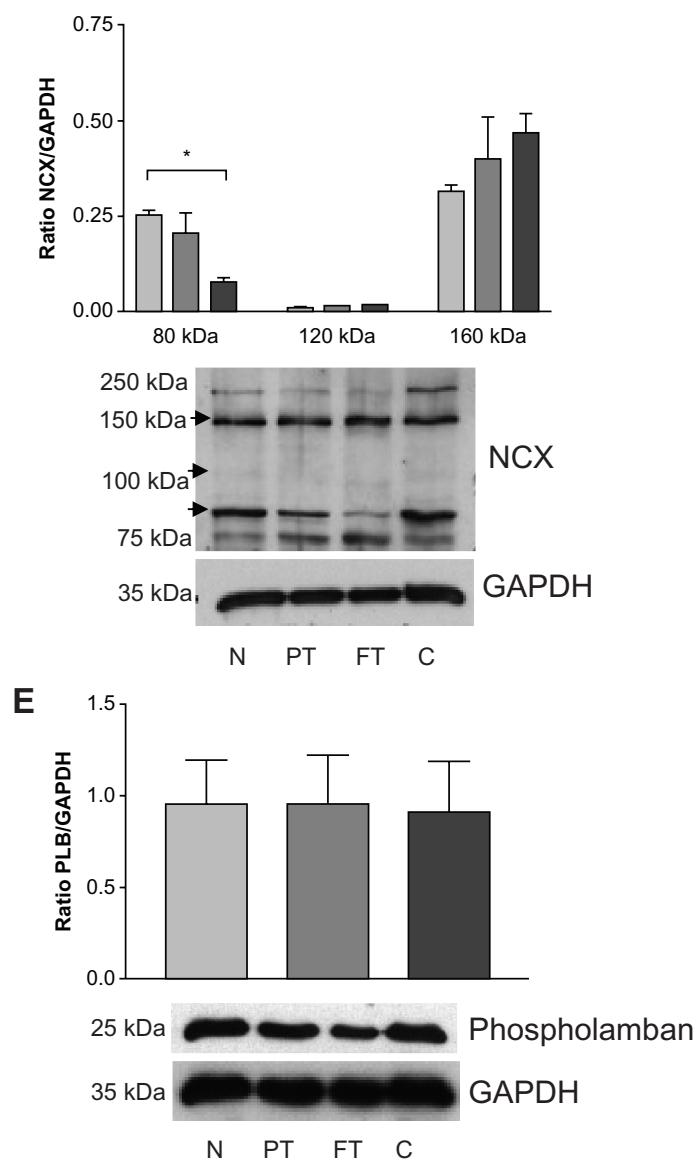

Figure 4 Western blot analysis of calcium regulatory proteins in the porcine left ventricle at 4 weeks post-Ml. For each protein, the autoradiograph identifies protein within the normal myocardium (N), FTMI, and PTMI from the same pig heart, with normal mouse myocardium (C) as a biological control. (A) Calcineurin expression (60 kDa) did not significantly alter; (B) SERCA expression ( $110 \mathrm{kDa}$ ) decreased slightly in PTMl yet was significantly lower in FTMI $(P=0.005)$; (C) Calsequestrin expression (60 kDa) was decreased in FTMI but this was not significant; (D) Expression of the $80 \mathrm{kDa}$ isoform of sodium-calcium exchanger was significantly decreased in FTMI, but no changes were observed across groups relating to expression of the $120 \mathrm{kDa}$ and $160 \mathrm{kDa}$ isoforms; and (E) Total phospholamban expression (25 kDa) did not change.

Note: $* *(P<0.01), *(P<0.05)$.

Abbreviations: C, normal mouse myocardium; N, normal myocardium; FTMI, full thickness infarct; PTMI, partial thickness infarct; SERCA, sarcoplasmic reticulum Ca ${ }^{2+}$ ATPase.

\section{Acknowledgments}

We are grateful to all staff of the BSU for their assistance and care of the animals used in this study. We thank Biocompatibles Ltd for provision of the embolization beads. EW is in receipt of a Medical Research Council CASE studentship.

\section{Disclosure}

The authors report no conflicts of interest in this work.

\section{References}

1. Hasenfuss G. Alterations of calcium-regulatory proteins in heart failure. Cardiovasc Res. 1998;37(2):279-289.

2. Hobai IA, O'Rourke B. Decreased sarcoplasmic reticulum calcium content is responsible for defective excitation-contraction coupling in canine heart failure. Circulation. 2001;103(11): $1577-1584$.

3. Sjaastad I, Wasserstrom JA, Sejersted OM. Heart failure - a challenge to our current concepts of excitation-contraction coupling. $J$ Physiol. 2003;546(Pt 1):33-47. 
4. Fallavollita JA, Canty JM Jr. Ischemic cardiomyopathy in pigs with twovessel occlusion and viable, chronically dysfunctional myocardium. Am J Physiol Heart Circ Physiol. 2002;282(4):H1370-H1379.

5. van der Velden J, Merkus D, Klarenbeek BR, et al. Alterations in myofilament function contribute to left ventricular dysfunction in pigs early after myocardial infarction. Circ Res. 2004;95(11):e85-e95.

6. Sabbah HN, Stein PD, Kono T, et al. A canine model of chronic heart failure produced by multiple sequential coronary microembolizations. Am J Physiol. 1991;260(4 Pt 2):H1379-H1384.

7. Munagala VK, Hart CY, Burnett JC Jr, Meyer DM, Redfield MM. Ventricular structure and function in aged dogs with renal hypertension: a model of experimental diastolic heart failure. Circulation. 2005;111(9): 1128-1135.

8. Brasselet C, Morichetti MC, Messas E, et al. Skeletal myoblast transplantation through a catheter-based coronary sinus approach: an effective means of improving function of infarcted myocardium. Eur Heart J. 2005;26(15):1551-1556.

9. Hasnat AK, van der Velde ET, Hon JK, Yacoub MH. Reproducible model of post-infarction left ventricular dysfunction: haemodynamic characterization by conductance catheter. Eur J Cardiothorac Surg. 2003;24(1):98-104.
10. De Scheerder I, Wang K, Wilczek K, et al. Experimental study of thrombogenicity and foreign body reaction induced by heparin-coated coronary stents. Circulation. 1997;95(6):1549-1553.

11. Kornowski R, Hong MK, Tio FO, Bramwell O, Wu H, Leon MB. In-stent restenosis: contributions of inflammatory responses and arterial injury to neointimal hyperplasia. J Am Coll Cardiol. 1998;31(1):224-230.

12. Malik N, Francis SE, Holt CM, et al. Apoptosis and cell proliferation after porcine coronary angioplasty. Circulation. 1998;98(16):1657-1665.

13. Malik N, Gunn J, Holt CM, et al. Intravascular stents: a new technique for tissue processing for histology, immunohistochemistry, and transmission electron microscopy. Heart. 1998;80(5):509-516.

14. Malik N, Gunn J, Shepherd L, Crossman DC, Cumberland DC, Holt CM. Phosphorylcholine-coated stents in porcine coronary arteries: in vivo assessment of biocompatibility. J Invasive Cardiol. 2001;13(3): 193-201.

15. Wright EJ, Farrell KA, Malik N, et al. Encapsulated glucagon-like peptide-1-producing mesenchymal stem cells have a beneficial effect on failing pig hearts. Stem Cells Transl Med. 2012;1(10):759-769.
Research Reports in Clinical Cardiology

\section{Publish your work in this journal}

Research Reports in Clinical Cardiology is an international, peerreviewed, open access journal publishing original research, reports, editorials, reviews and commentaries on all areas of cardiology in the clinic and laboratory. The manuscript management system is completely online and includes a very quick and fair peer-review system.

\section{Dovepress}

Visit http://www.dovepress.com/testimonials.php to read real quotes from published authors. 